\title{
Effectivity of Psychotherapy Interventions for Anxiety in Medical Students: A Systematic Review
}

\author{
Andrian Fajar Kusumadewi ${ }^{1 *}$, Carla Raymondalexas Marchira ${ }^{1}$, Widyandana Widyandana $^{2}$, Ronny Tri Wirasto ${ }^{1}$ \\ ${ }^{1}$ Department of Psychiatry, Faculty of Medicine, Public Health and Nursing, Universitas Gadjah Mada, Dr Sardjito General \\ Hospital, Yogyakarta, Indonesia; ${ }^{2}$ Department of Medical Education and Bioethics, Faculty of Medicine, Public Health and \\ Nursing, Universitas Gadjah Mada, Dr Sardjito General Hospital, Yogyakarta, Indonesia
}

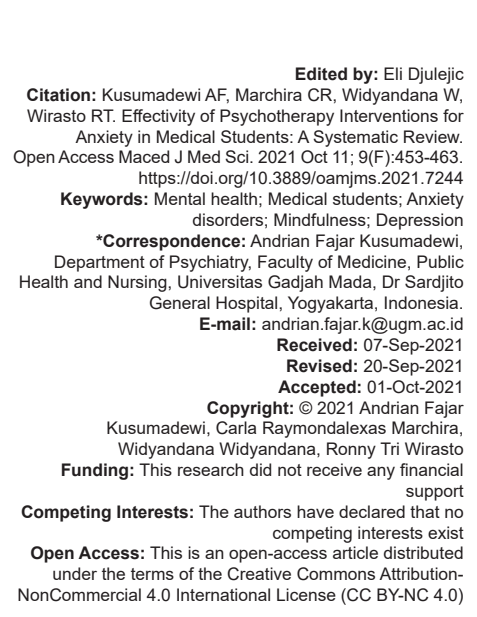

\section{Abstract}

BACKGROUND: The mental health of medical students has long been a topic of concern in many countries. Much research in Europe reported that around 30\% of medical students suffered from anxiety. Anxiety disorders have significant physical and emotional consequences. Various studies show that excessive, unmanaged stress related to poor academic performance in medical students leads to cynical personalities, lack of empathy, and suicidal ideas.

AIM: This study aimed to identify studies for psychotherapy interventions carried out in medical students and analyze each impact on anxiety level in medical students.

METHODS: This systematic review was conducted with evidence sourced from 2000 to 2020 . The review process followed the Preferred Reporting Items for Systematic Reviews and Meta-Analyses guidelines. The study was registered with the Prospective Registering of Systematic Reviews database (Protocol ID: CRD42020180650).

RESULTS: Twenty-three studies meet the inclusion criteria, and there are various psychotherapy interventions to deal with anxiety. Most studies reported that students who received mindfulness-based interventions reported lower anxiety, depression, and stress.

CONCLUSION: There are various interventions carried out to decrease stress levels, depression, and anxiety in medical students. The most effective psychotherapy was found in cognitive and behavior intervention and mindfulness intervention.

\section{Introduction}

The mental health of medical students has long been a topic of concern in many countries. Medical students have significant responsibilities and demands related to their future profession. Being a doctor is famous for having very little room for error because the profession itself is closely related to life and death. Therefore, medical students are expected to have adequate knowledge and the appropriate skill set to give the best medical service possible. The amount of detailed materials to cover and master in such a short time causes anxiety in medical students [1].

Medical students are more prone to anxiety than non-medical students and the general population [2], [3]. Various researches in Europe reported that around 30\% of medical students suffered from either depression or anxiety. This corresponds to research conducted in Brazil, where it was found that $20-50 \%$ of medical students have mood disorders [4], [5]. Signs of anxiety increased in 1st-year medical students and decreased through the 4th year but increased again in the 5th year of medical education [6]. Other research reported that $11.5 \%$ of medical students suffer from anxiety during medical education [7]. Frequently found symptoms are restlessness, fatigue, difficulty concentrating, mindlessness, irritability, feeling tense, and sleeping disturbance [8].

Rigid personal standards trigger anxiety in medical students to achieve high targets, academic burden, lack of sleep, financial burden, and exposure to patient's suffering and death. Students in medical school have tendencies to have neurotic and perfectionist personalities, which are a predisposition to anxiety [9]. As a medical student, someone is required to be prepared for several consequences, such as a long education process, academic tasks that require responsibility and commitment, competitive and less than ideal learning environment, and lack of sleep and social engagement that precipitates anxiety [10].

Anxiety results in significant consequences physically, emotionally, mentally, and professionally. Various studies show excessive, unmanaged stress related to poor academic performance, medical students with cynical personalities, lack of empathy, and lack of ability to care for patients. Patient care quality by doctors with anxiety also shows poor efficiency. Medical 
students with anxiety may lose academic integrity, academic dishonesty, career dissatisfaction, alcohol or substance dependency, and suicide [11].

Intervention in anxiety consists of pharmacotherapy and non-pharmacotherapy. The most common therapy for anxiety in medical students is psychotherapy. There has been various research about psychotherapy intervention against anxiety in medical students, such as cognitive-behavior therapy, mindfulness, psychoeducation, supportive therapy, yoga, and stress management [12], [13].

In the previous studies that have been established that psychotherapy interventions are essential in reducing anxiety in medical students, little remains to be known about which interventions are the most effective and feasible to be applied. This systematic review aims to identify studies for psychotherapy interventions in medical students to analyze each impact on anxiety level in medical students.

\section{Methods}

The systematic review of the literature was conducted with evidence sourced from 2000 to 2020. The review process followed the Preferred Reporting Items for Systematic Reviews and MetaAnalyses guidelines. The study was registered with the Prospective Registering of Systematic Reviews database (Protocol ID: CRD42020180650).

\section{criteria}

\section{Literature search strategy and selection}

We searched the following electronic databases: Cochrane Library, PubMed, ScienceDirect, EBSCOhost, Scopus, Medline, ProQuest, and CENTRAL (Cochrane Controlled Register of Trials) for randomized controlled trial (RCT) or quasi-experimental articles reporting psychotherapy interventions for anxiety in medical students. The search of the key terms was performed using OR and AND functions and database-specific filters were also used. Articles were identified with titles or abstracts that included terms or variants of the following groups: ("medical student*" OR "clinical clerkship" OR "student doctor*") AND ("anxiety" OR "anxiety disorder*) AND ("RCT" OR "quasi" OR "clinical trial" OR "RCT"). The search was limited to articles published between 2000 and 2020. The reference lists of the included articles were also screened manually to identify additional studies.

Search results were exported into Microsoft Excel and duplicates were removed before titles and abstracts were screened to the inclusion or exclusion criteria. Relevant titles and abstracts would then be going through a full-text reading and further review.
The studies were included only if they were: Discussing anxiety intervention on medical students, psychological intervention, either RCT or quasi-experimental clinical trial, published between 2000 and 2020, and written in English. We excluded: Articles including either residents or postgraduate medical students as subjects, pharmacological intervention as the control group, and the participant had already been diagnosed with anxiety disorder and taken care of by a professional during the study period.

\section{Data extraction}

Citations were screened by seven reviewers and were checked independently by the 4 other reviewers. All reviewers confirm the eligibility of the identified studies. Any disagreements about possible inclusion were resolved by a group discussion. Data were collected and summarized in a standardized data extraction form, reporting the aim of the study, study design, number and characteristic of participants, type and description of psychotherapy interventions, duration of follow-up, outcome, and study results. The search process is shown in Figure 1. A narrative synthesis was arranged because the studies were different in terms of design, setting, interventions, and outcome measures [14].

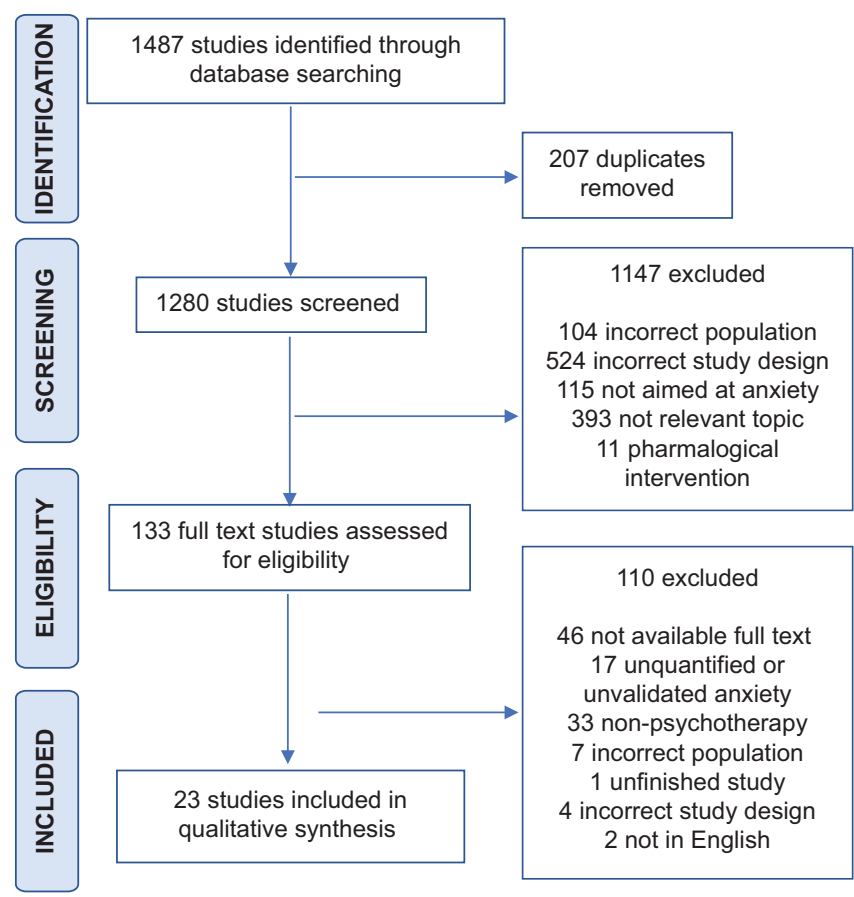

Figure 1: Summary of the search and exclusion processes

\section{Risk of bias assessment}

Each randomized study was assessed for bias using the Cochrane Risk of Bias 2 (RoB 2) tool and Cochrane Robins-I tool for quasi-experimental studies. The risk of bias tool assesses several domains about the randomization process, selection of participants, confounding factors, classification of interventions, 
deviations from the intended interventions, missing outcome data, measurement of the outcomes, and selection of reported results. The risk of bias in each subcategory was classified as high, low, or some concerns. The assessment of bias was conducted independently by two authors and decisions were compared and discussed to achieve consensus.

\section{Results}

\section{Characteristics of the included studies}

of the included studies. Four studies were conducted in America [15], [16], [17], [18], three in Malaysia [19], [20], [21], three in India [22], [23], [24], three in China [25], [26], [27], two in Australia [28], [29], two in Brazil [30], [31], one in Switzerland [32], one in Germany [33], one in Canada [34], Thailand [35], and one more in Iran [36].

Most of the studies were aimed at the 1st to 3 rd year of the preclinical students. One study included medical students in their final 2 years of study [29]. Two studies included students in the 5th year of medical studies [25], [35], and four included randomly selected undergraduate medical students [22], [24], [26], [36]. Studies explained in this review used various psychotherapy techniques to reduce anxiety levels in medical students, including mindfulness-based interventions, psychoeducation and relaxation techniques, cognitive and behavioral interventions, hypnosis, and life skill training.

\section{studies \\ Methodological quality of the included}

Among all studies included in the review, there were 12 RCTs [15], [16], [17], [18], [19], [20], [21], [22], [23], [24], [25], [26], [27], [28], [29], [30], [31], [32], [33], [34], [35], [36] and nine quasi-experimental studies [16], [17], [18], [18], [19], [22], [23], [30], [32], [36]. The risk of bias of the randomized studies based on the RoB-2 tool is reported in Table 2 . In the 13 journals of randomized studies, 12 were judged to have a low risk of bias, and the remaining ones were judged as have some concerns regarding bias. The risk of bias that most often arises is in the measurement of outcome bias because there is no information on whether the subject knows the results of the studies. Nine studies were screened for risk of bias based on the Robins-I tool reported in Table 3. The overall quality of the studies has a low risk of bias (overall results based on the risk of bias analysis). In a study, there was a possibility of bias because it was not possible to determine how many participants. Another study found bias due to missing data in the middle of the research, so it had an attrition rate of $20 \%$. In addition, the study did not provide information on whether there were deviations from the intervention data, bias in measuring outcome, and bias in the selection of reported outcomes.

\section{Description of the psychotherapy interventions for anxiety}

\section{Mindfulness-based interventions}

Eight studies reported mindfulness-based interventions [15], [17], [18], [28], [29], [31], [35], [34]. The core of these interventions is to emphasize mindfulness in medical students through several courses, lessons programs (pieces of training such as Kabat-Zinn program), peer support program, meditation including Buddhist Anapanasati, eating, and walking meditation, body scan, breath awareness, mindful stretching (Hatha Yoga), progressive relaxation, guided imagery, shorter version of MBSM, MMP based on the principles of the 8-week MBSR and MBCT programs, and also a mindfulness practice guided by audio compact disc or DVD (MindfulGym DVD). Students were taught a variety of mindfulness techniques to focus their attention on the present-moment experience, decrease emotional reactivity, increase feelings of gratitude, manage fatigue and anxiety, have work-life balance, be aware of the breath mindfully, deal with patient suffering, deal with the need to be perfect, and make a point of self-acceptance. The duration of these interventions ranged from 4 to 22 weeks.

\section{Psychoeducation and relaxation technique}

Six studies reported psychoeducation and relaxation techniques as psychotherapy interventions for anxiety in medical students. The participants were given an educational program about simple relaxation, PMRT, systematic desensitization, Yoga session, or combined technique. One study used a specific yoga called super brain yoga [22] to evaluate reducing anxiety levels and autonomic functions. Counseling or psychoeducation sessions focused on education about psychological and physical symptoms of test anxiety and the consequences of low marks, pessimistic thoughts about themselves and scholastic performance [21]. Participants were also encouraged to write the hierarchy of least to most anxiety-provoking situations and describe the feelings and emotions related to items in the list. In systematic desensitization, participants were advised to practice relaxation therapy and imagine the minor level of an anxiety-producing situation in the hierarchy. If participants felt tension and anxiety, they were advised to stop and return to the relaxation technique until they were comfortable. Once again, after they relaxed, they were counseled to continue to imagine the anxiety-producing situation. In another study [33], participants received 1-hour psychoeducation delivered by a psychologist. Several issues were addressed, such as emotional reactions toward stressors, unconscious 
Table 1: Characteristics of the included study

\begin{tabular}{|c|c|c|c|c|c|c|}
\hline Authors & $\begin{array}{l}\text { Subject } \\
\text { experimental/ } \\
\text { control group }\end{array}$ & Training level & Assessment methods and tools & Intervention & $\begin{array}{l}\text { Intervention duration } \\
\text { and frequency }\end{array}$ & Results \\
\hline Bansal et al., [23] & $90 / 0$ & $\begin{array}{l}\text { Third-semester medical } \\
\text { students in department } \\
\text { of community medicine }\end{array}$ & $\begin{array}{l}\text { GHQ-28 (28-item General Health } \\
\text { Questionnaire) }\end{array}$ & Yoga & $\begin{array}{l}\text { 45-min yoga session } \\
\text { daily for } 1 \text { month }\end{array}$ & $\begin{array}{l}\text { Students reported significant } \\
\text { improvement in somatic symptoms, } \\
\text { anxiety, social dysfunction and } \\
\text { depression }\end{array}$ \\
\hline $\begin{array}{l}\text { Bermudez } \\
\text { et al. [30] }\end{array}$ & $62 / 0$ & $\begin{array}{l}\text { Over } 18 \text { year old of age } \\
\text { in the second semester } \\
\text { of medical school }\end{array}$ & $\begin{array}{l}\text { MEAQ (Multidimensional } \\
\text { Experiential Avoidance Question); } \\
\text { Q-LES Q-SF (Quality of Life } \\
\text { [QoL] Enjoyment and Satisfaction } \\
\text { Questionnaire); The Rosenberg } \\
\text { Self-Esteem Scale (RSE); The } \\
\text { Interpersonal Reactivity Index (IRI); } \\
\text { The Overall Anxiety Severity and } \\
\text { Impairment Scale (OASIS); Beck } \\
\text { Depression Inventory (BDI) }\end{array}$ & $\begin{array}{l}\text { Single-session } \\
\text { preventive } \\
\text { intervention using } \\
\text { Unified Protocol } \\
\text { for Transdiagnostic } \\
\text { Treatment of } \\
\text { Emotional Disorders }\end{array}$ & $\begin{array}{l}\text { A single 2-hour } \\
\text { session }\end{array}$ & $\begin{array}{l}\text { The intervention led to significant } \\
\text { reduction in number of student } \\
\text { having social anxiety disorder and } \\
\text { panic disorder, and significant } \\
\text { reduction in depressive symptoms }\end{array}$ \\
\hline Brennan et al. [16] & $42 / 0$ & $1^{\text {st }}$ year medical students & $\begin{array}{l}\text { BDI-II (Beck Depression } \\
\text { Inventory-II); } \\
\text { Beck Anxiety Inventory (BAI); } \\
\text { SRRS-S (Social Readjustment } \\
\text { Rating Scale-Revised); CSE (Coping } \\
\text { Self Efficacy Scale) }\end{array}$ & $\begin{array}{l}\text { Relaxation } \\
\text { techniques }\end{array}$ & $\begin{array}{l}8 \text { sessions }(4 \\
\text { sessions per } \\
\text { semester) over } 9 \\
\text { months }\end{array}$ & $\begin{array}{l}\text { Students reported significant } \\
\text { improvement in anxiety symptoms } \\
\text { and self-efficacy but no improvement } \\
\text { in depression symptoms }\end{array}$ \\
\hline $\begin{array}{l}\text { Danilewitz } \\
\text { et al. [34] }\end{array}$ & $30 / 0$ & $\begin{array}{l}\text { First and } 2^{\text {nd }} \text { year } \\
\text { medical students }\end{array}$ & $\begin{array}{l}\text { Depression Anxiety and Stress } \\
\text { Scale; Jefferson Scale of Physician } \\
\text { Empathy -Student Version; Five } \\
\text { Facets of Mindfulness Questionnaire } \\
\text { (FFMQ); Self- Compassion Scale } \\
\text { (SCS); Adapted Altruism Scale }\end{array}$ & $\begin{array}{l}\text { Peer-led Mindfulness } \\
\text { Meditation Program } \\
\text { (MMP) }\end{array}$ & $\begin{array}{l}\text { 1-1, 5-h weekly } \\
\text { sessions for } 8 \text { weeks }\end{array}$ & $\begin{array}{l}\text { Students reported significant } \\
\text { changes in level of stress, } \\
\text { self-compassion, altruism, and two } \\
\text { facets of mindfulness (describe and } \\
\text { non-react) }\end{array}$ \\
\hline $\begin{array}{l}\text { Finkelstein } \\
\text { et al. [17] }\end{array}$ & $\begin{array}{l}\text { Time 1:72 } \\
\text { Time 2:51 } \\
\text { Time 3:63 }\end{array}$ & $2^{\text {nd }}$ year medical students & $\begin{array}{l}\text { SCL-90 Anxiety Subscale; POMS; } \\
\text { 2-Item } \\
\text { Depression Index; PSMS }\end{array}$ & $\begin{array}{l}\text { Mindfulness Elective } \\
\text { Program }\end{array}$ & $\begin{array}{l}10 \text { weekly } 2-\mathrm{h} \\
\text { sessions }\end{array}$ & $\begin{array}{l}\text { Students reported significant } \\
\text { decrease in anxiety level and } \\
\text { perceived stress over the } \\
\text { intervention course and the decrease } \\
\text { was sustained for } 3 \text { months }\end{array}$ \\
\hline $\begin{array}{l}\text { Gurupriya and } \\
\text { Mathangi [22] }\end{array}$ & $32 / 0$ & $\begin{array}{l}\text { Undergraduate medical } \\
\text { students within the age } \\
\text { range of } 19-21 \text { years }\end{array}$ & $\begin{array}{l}\text { Generalized Anxiety Disorder } \\
\text { questionnaire (GAD-7) }\end{array}$ & Super brain yoga & $\begin{array}{l}15-20 \text { min daily for } \\
30 \text { days }\end{array}$ & $\begin{array}{l}\text { Students reported significant } \\
\text { decrease in anxiety level after } 30 \\
\text { days of intervention }\end{array}$ \\
\hline Hammer et al. [32] & 19/17 & $1^{\text {st }}$-year medical students & $\begin{array}{l}\text { Prüfungsangstfragebogen } \\
\text { (PAF) - German version of TAI-G } \\
\text { questionnaire }\end{array}$ & Clinical hypnosis & $\begin{array}{l}1 \mathrm{~h} \text { of hypnosis } \\
\text { session, followed by } \\
\text { listening to the audio } \\
\text { record daily until } \\
\text { examination days }\end{array}$ & $\begin{array}{l}\text { Students reported reduced global } \\
\text { test anxiety (PAF) and lack of } \\
\text { confidence scores after intervention, } \\
\text { although there was no statistically } \\
\text { significant difference in test anxiety } \\
\text { scores between intervention and } \\
\text { control group }\end{array}$ \\
\hline Lattie et al. [19] & $53 / 0$ & $1^{\text {st }}$-year medical students & $\begin{array}{l}\text { Medical Student Well-Being } \\
\text { Index (MSWBI); Perceived Stress } \\
\text { Scale (PSS); Cognitive and } \\
\text { Behavioral Response to Stress } \\
\text { Scale (CB-RSS); Patient Health } \\
\text { Questionnaire-8 (PHQ-8); GAD-7 }\end{array}$ & $\begin{array}{l}\text { Internet-based } \\
\text { cognitive-behavioral } \\
\text { therapy (CBT) }\end{array}$ & 16 weeks & $\begin{array}{l}\text { Self-reported symptoms of } \\
\text { depression and anxiety were low at } \\
\text { baseline, and continued to be low } \\
\text { at the end of the program. Slight } \\
\text { increases were observed from } \\
\text { baseline to end of program in the } \\
\text { self-reported use of cognitive coping } \\
\text { skills }\end{array}$ \\
\hline Li et al. [25] & $29 / 26$ & $\begin{array}{l}\text { Ninth- semester medical } \\
\text { students }\end{array}$ & $\begin{array}{l}\text { Chinese version of Self-Rating } \\
\text { Anxiety Scale (SAS); Self-Rating } \\
\text { Depression Scale (SDS); Trait } \\
\text { Coping Style Questionnaire (TCSQ); } \\
\text { interpersonal Support Evaluation List } \\
\text { (ISEL); Self-Esteem Scale (SES) }\end{array}$ & $\begin{array}{l}\text { Williams LifeSkills } \\
\text { Training (WLST) }\end{array}$ & $\begin{array}{l}\text { 2-h weekly sessions } \\
\text { over } 8 \text { weeks }\end{array}$ & $\begin{array}{l}\text { Students reported decreased anxiety } \\
\text { and depression, as well as increased } \\
\text { self-esteem and social support }\end{array}$ \\
\hline McGrady et al. [15] & $171 / 278$ & $1^{\text {st }}$-year medical students & $\begin{array}{l}\text { BDI-II (Beck Depression Inventory); } \\
\text { BAI; SRRS-R (Social Readjustment } \\
\text { Rating Scale Revised); Brief } \\
\text { questionnaire indicating frequency of } \\
\text { acute illness }\end{array}$ & $\begin{array}{l}\text { Stress management } \\
\text { and wellness } \\
\text { program }\end{array}$ & $\begin{array}{l}45-\text { min sessions } \\
\text { twice a month for } 4 \\
\text { consecutive months }\end{array}$ & $\begin{array}{l}\text { Students reported significant } \\
\text { decrease in depression after } \\
\text { intervention although there were } \\
\text { no significant changes in anxiety or } \\
\text { frequency of acute illness }\end{array}$ \\
\hline Moir et al. [28] & $111 / 121$ & $\begin{array}{l}\text { Second- and 3rd year } \\
\text { medical students }\end{array}$ & $\begin{array}{l}\text { Primary Health Questionnaire } \\
\text { (PHQ-9); GAD-7; QoL (Linear } \\
\text { Analogue Self-Assessment [LASA]; } \\
\text { Resilience (25-item questionnaire); } \\
\text { Academic self-concept (Perceived } \\
\text { Competence Scale); Academic } \\
\text { motivation (the Motivated Strategies } \\
\text { for Learning Questionnaire) }\end{array}$ & $\begin{array}{l}\text { Peer support } \\
\text { and peer-taught } \\
\text { mindfulness practice }\end{array}$ & $\begin{array}{l}24 \mathrm{~h} \text { peer leaders' } \\
\text { training over } 8 \\
\text { weeks; weekly } \\
\text { mindfulness practice } \\
\text { led by peer leaders } \\
\text { for } 25 \text { consecutive } \\
\text { weeks }\end{array}$ & $\begin{array}{l}6 \text { months after intervention, students } \\
\text { in the intervention group did not gain } \\
\text { significant improvement in anxiety } \\
\text { and depression symptoms as well as } \\
\text { in the QoL, resilience or academic } \\
\text { measures }\end{array}$ \\
\hline $\begin{array}{l}\text { Dehghan-Nayeri } \\
\text { et al., [36] }\end{array}$ & $100 / 100$ & $\begin{array}{l}\text { Female dormitory } \\
\text { medical students }\end{array}$ & $\begin{array}{l}\text { The Spielberger anxiety } \\
\text { inventory; Modified version of the } \\
\text { WHOQOL-100 (WHO quality of life } \\
\text { questionnaire) }\end{array}$ & $\begin{array}{l}\text { Relaxation } \\
\text { techniques }\end{array}$ & $\begin{array}{l}8 \text { weeks; } 15-20 \text { min } \\
\text { daily }\end{array}$ & $\begin{array}{l}8 \text { weeks after intervention, } \\
\text { experimental group reported a } \\
\text { reduction in anxiety level and } \\
\text { subsequently, an improvement in } \\
\text { QoL }\end{array}$ \\
\hline
\end{tabular}


Table 1: (Continued)

\begin{tabular}{|c|c|c|c|c|c|c|}
\hline Authors & $\begin{array}{l}\text { Subject } \\
\text { experimental/ } \\
\text { control group }\end{array}$ & Training level & Assessment methods and tools & Intervention & $\begin{array}{l}\text { Intervention duration } \\
\text { and frequency }\end{array}$ & Results \\
\hline Neto et al. [31] & $70 / 71$ & $1^{\text {st }}$ year medical students & $\begin{array}{l}\text { Duke Religion Index validated for use } \\
\text { in Brazil; Depression, Anxiety, and } \\
\text { Stress Scale } 21 \text { (DASS-21): validated } \\
\text { for Portuguese; WHOQOL-BREF } \\
\text { (World Health Organization QoL: } \\
\text { validated for Portuguese); Five } \\
\text { Facets of Mindfulness (FFMQ-BR); } \\
\text { Subjective questionnaire yes/no }\end{array}$ & $\begin{array}{l}\text { Mindfulness } \\
\text { meditation }\end{array}$ & $\begin{array}{l}\text { 2-h sessions for } 6 \\
\text { consecutive weeks }\end{array}$ & $\begin{array}{l}\text { Students undergoing large-group } \\
\text { mindfulness meditation did not } \\
\text { experience significant improvement } \\
\text { in QoL, anxiety, stress and } \\
\text { depression }\end{array}$ \\
\hline Paholpak et al. [35] & $30 / 28$ & $5^{\text {th }}$ year medical students & $\begin{array}{l}\text { Self-Checklists-90 (SCL-90); } \\
\text { Wechsler Memory Scale form } \\
\text { I (WMS-I); Raven's Advanced } \\
\text { Progressive Matrices (APM); } \\
\text { psychiatry course examination score }\end{array}$ & $\begin{array}{l}\text { Buddhist } \\
\text { Anapanasati } \\
\text { Meditation (Mindful } \\
\text { awareness) }\end{array}$ & $\begin{array}{l}\text { 20-min session daily } \\
\text { for } 28 \text { days }\end{array}$ & $\begin{array}{l}\text { After } 4 \text { weeks of intervention, } \\
\text { students did not experience changes } \\
\text { in psychiatric symptoms, memory } \\
\text { function, intellectual performance } \\
\text { and academic achievement }\end{array}$ \\
\hline Phang et al., [20] & $37 / 38$ & $\begin{array}{l}\text { medical students in year } \\
1 \text { to } 3 \text { of studies }\end{array}$ & $\begin{array}{l}\text { mindful attention awareness scale } \\
\text { (MAAS), Perceived stress scale } \\
\text { (PSS), general health questionnaire } \\
\text { (GHQ), general self-efficacy (GSE) }\end{array}$ & Mindfulness & $\begin{array}{l}\text { Once a week at } \\
2 \mathrm{~h} \text { session for } 5 \\
\text { consecutive weeks } \\
\text { of shorter version of } \\
\text { mindfullness based } \\
\text { stress management } \\
\text { (MBSM) adapted } \\
\text { from 8-week MBSR } \\
\text { and MBCT program }\end{array}$ & $\begin{array}{l}\text { Significant reduction for } 1 \text { week } \\
\text { post-intervention in perceived } \\
\text { stress score }(p=0.009) \text { and mental } \\
\text { distress score }(p=0.003) \text {, with } \\
\text { improvemnet in mindfullness } \\
(p=0.40) \text { and self-efficacy score } \\
(p<0.001) \text {. After } 6 \text { months, } \\
\text { significant increase in self efficacy } \\
\text { scores is maintained, but other } \\
\text { scores were not found to be } \\
\text { significant }\end{array}$ \\
\hline $\begin{array}{l}\text { Rosenzweig } \\
\text { et al. [18] }\end{array}$ & $140 / 162$ & $\begin{array}{l}2^{\text {nd }} \text {-year medical } \\
\text { students }\end{array}$ & The Profile of Mood States (POMS) & $\begin{array}{l}\text { Mindfulness-Based } \\
\text { Stress (MBSR) } \\
\text { course }\end{array}$ & $\begin{array}{l}90 \text {-min weekly } \\
\text { seminars over } 10 \\
\text { weeks; } 20 \text {-min } \\
\text { meditation for } 6 \text { days } \\
\text { a week }\end{array}$ & $\begin{array}{l}\text { Students reported lower Total Mood } \\
\text { Disturbance (TMD) scores and were } \\
\text { able to manage stress better as } \\
\text { shown through better tension-anxiety, } \\
\text { confusion-bewilderment, } \\
\text { fatigue-inertia, and vigor-activity } \\
\text { scores }\end{array}$ \\
\hline $\begin{array}{l}\text { Saravanan and } \\
\text { Kingston [21] }\end{array}$ & $32 / 33$ & $1^{\text {st }}$-year medical students & $\begin{array}{l}\text { Westside test anxiety scale (WTAS); } \\
\text { The Kessler Perceived Distress } \\
\text { Scale (K-10); Academic Motivation } \\
\text { Scale (AMS) }\end{array}$ & $\begin{array}{l}\text { Psychoeducation, } \\
\text { Progressive } \\
\text { Muscular Relaxation } \\
\text { Therapy (PMRT), } \\
\text { and systematic } \\
\text { desensitization }\end{array}$ & $\begin{array}{l}5 \text { sessions over a } \\
\text { period of } 3 \text { weeks }\end{array}$ & $\begin{array}{l}\text { Students reported reduction in } \\
\text { anxiety, psychological distress, and } \\
\text { amotivation, as well as increased } \\
\text { intrinsic and extrinsic motivation after } \\
\text { intervention }\end{array}$ \\
\hline $\begin{array}{l}\text { Velayudhan } \\
\text { et al. [24] }\end{array}$ & $60 / 60$ & Medical students & $\begin{array}{l}\text { BAI; Beck Depression inventory } \\
\text { (BDI) }\end{array}$ & $\begin{array}{l}\text { Counselling, } \\
\text { relaxation } \\
\text { techniques and } \\
\text { meditation }\end{array}$ & 1 month & $\begin{array}{l}\text { Students reported lower level } \\
\text { of anxiety and depression after } \\
\text { counseling }\end{array}$ \\
\hline Warnecke et al. [29] & $24 / 32$ & $\begin{array}{l}\text { Medical students who } \\
\text { are in their final } 2 \text { years }\end{array}$ & $\begin{array}{l}\text { Perceived stress scale (PSS); } \\
\text { Depression, Anxiety and Stress } \\
\text { Scale (DASS) }\end{array}$ & $\begin{array}{l}\text { Audio } C D \text {-guided } \\
\text { mindfulness practice }\end{array}$ & $\begin{array}{l}\text { 30-min daily session } \\
\text { for } 8 \text { weeks }\end{array}$ & $\begin{array}{l}\text { Students reported significant } \\
\text { decrease in stress and anxiety level } \\
\text { after } 8 \text { weeks of intervention. This } \\
\text { effect was also maintained after } 8 \\
\text { weeks of intervention }\end{array}$ \\
\hline Xu et al. [26] & $25 / 23$ & Medical students & $\begin{array}{l}\text { Satisfaction with life scale (SWLS); } \\
\text { Positive affect and Negative Affect } \\
\text { Schedule (PANAS); Center for } \\
\text { Epidemiological Studies Depression } \\
\text { Scale (CES-D); State Anxiety } \\
\text { inventory (SAI) }\end{array}$ & $\begin{array}{l}\text { Cognitive (Positive } \\
\text { Psychology } \\
\text { Interventions) }\end{array}$ & $\begin{array}{l}\text { Once a week for } 10 \\
\text { weeks }\end{array}$ & $\begin{array}{l}\text { Students reported larger } \\
\text { improvement in subjective well-being } \\
\text { (SWB), greater relief in depression } \\
\text { and anxiety after intervention } \\
\text { compared to control group }\end{array}$ \\
\hline Xu et al. [27] & $39 / 48$ & $1^{\text {st }}$ year medical students & $\begin{array}{l}\text { Chinese version of Ryff's scales of } \\
\text { psychological well-being (RPWB); } \\
\text { Chinese college student adjustment } \\
\text { scale (CCSAS); Chinese version } \\
\text { of the centre for epidemiological } \\
\text { studies depression scale (CES-D); } \\
\text { Chinese version of the SAI }\end{array}$ & Well-being therapy & $\begin{array}{l}\text { 2-h session } \\
\text { every week for } 5 \\
\text { consecutive weeks }\end{array}$ & $\begin{array}{l}\text { Immediately after the intervention, } \\
\text { students were able to adapt better } \\
\text { to medical school. } 3 \text { months after } \\
\text { intervention, students reported } \\
\text { improved psychological well-being } \\
\text { and reduced symptoms of anxiety } \\
\text { and depression }\end{array}$ \\
\hline
\end{tabular}

Table 2: Cochrane risk of bias 2 (RoB-2) tool

\begin{tabular}{|c|c|c|c|c|c|c|c|}
\hline Study & $\begin{array}{l}\text { Risk of bias } \\
\text { arising from the } \\
\text { randomization } \\
\text { process }\end{array}$ & $\begin{array}{l}\text { Risk of bias due to } \\
\text { deviations from the intended } \\
\text { interventions (effect of } \\
\text { assignment to intervention) }\end{array}$ & $\begin{array}{l}\text { Risk of bias due to } \\
\text { deviations from the intended } \\
\text { interventions (effect of } \\
\text { adhering to intervention) }\end{array}$ & $\begin{array}{l}\text { Missing } \\
\text { outcome data }\end{array}$ & $\begin{array}{l}\text { Risk of bias in } \\
\text { measurement of the } \\
\text { outcome }\end{array}$ & $\begin{array}{l}\text { Risk of bias in selection } \\
\text { of the reported result }\end{array}$ & $\begin{array}{l}\text { Overall risk of } \\
\text { bias }\end{array}$ \\
\hline Danilewitz et al. [34] & Some concerns & Low risk & Low risk & Some concerns & Low risk & Low risk & Low risk \\
\hline Kötter and Niebuhr [33] & Low risk & Low risk & Low risk & Low risk & Low risk & Low risk & Low risk \\
\hline Li et al. [25] & Low risk & Low risk & Low risk & Low risk & Low risk & Low risk & Low risk \\
\hline McGrady et al. [15] & Low risk & Low risk & Low risk & Low risk & Low risk & Low risk & Low risk \\
\hline Moir et al. [28] & Low risk & Low risk & Low risk & Some concerns & Low risk & Low risk & Low risk \\
\hline Neto et al. [31] & Some concerns & Some concerns & Low risk & Low risk & Some concerns & Low risk & Some concerns \\
\hline Paholpak et al. [35] & Low risk & Low risk & Low risk & Low risk & Low risk & Low risk & Low risk \\
\hline Phang et al. [20] & Low risk & Low risk & Low risk & Low risk & Low risk & Low risk & Low risk \\
\hline Saravanan and & Low risk & Low risk & Low risk & Low risk & Low risk & Low risk & Low risk \\
\hline \multicolumn{8}{|l|}{ Kingston [21] } \\
\hline Xu et al. [27] & Low risk & Low risk & Low risk & Low risk & Low risk & Low risk & Low risk \\
\hline Xu et al. [26] & Low risk & Low risk & Low risk & Some concerns & Low risk & Low risk & Low risk \\
\hline Velayudhan et al. [24] & Low risk & Low risk & Low risk & Low risk & Low risk & Low risk & Low risk \\
\hline Warnecke et al. [29] & Low risk & Low risk & Low risk & Some concerns & Low risk & Low risk & Low risk \\
\hline
\end{tabular}


Table 3: Cochrane Robins-I tool

\begin{tabular}{|c|c|c|c|c|c|c|c|}
\hline Study & $\begin{array}{l}\text { Bias due to } \\
\text { confounding }\end{array}$ & $\begin{array}{l}\text { Bias in selection of } \\
\text { participants into the study }\end{array}$ & $\begin{array}{l}\text { Bias in classification } \\
\text { of interventions }\end{array}$ & $\begin{array}{l}\text { Bias due to deviations } \\
\text { from intended } \\
\text { interventions }\end{array}$ & Bias due to missing data & $\begin{array}{l}\text { Bias in } \\
\text { measurement of } \\
\text { outcomes }\end{array}$ & $\begin{array}{l}\text { Bias in } \\
\text { selection of the } \\
\text { reported result }\end{array}$ \\
\hline Bansal et al. [23] & Low risk & Low risk & Low risk & Low risk & Low risk & Low risk & Low risk \\
\hline $\begin{array}{l}\text { Bermudez } \\
\text { et al. [30] }\end{array}$ & Low risk & Low risk & Low risk & Low risk & Low risk & Low risk & Low risk \\
\hline Brennan et al. [16] & Low risk & $\begin{array}{l}\text { Moderate risk (but we cannot } \\
\text { determine how many students } \\
\text { attended the Resource Fair. } \\
\text { Forty-two students signed the } \\
\text { consent form. ) }\end{array}$ & Low risk & Low risk & Low risk & Low risk & Low risk \\
\hline $\begin{array}{l}\text { Dehghan-Nayeri } \\
\text { et al. [36] }\end{array}$ & Low risk & Low risk & Low risk & Low risk & Low risk & Low risk & Low risk \\
\hline $\begin{array}{l}\text { Finkelstein } \\
\text { et al. [17] }\end{array}$ & Low risk & Low risk & Low risk & Low risk & Low risk & Low risk & Low risk \\
\hline $\begin{array}{l}\text { Gurupriya and } \\
\text { Mathangi [22] }\end{array}$ & Low risk & Low risk & Low risk & Low risk & $\begin{array}{l}\text { Moderate Risk } \\
\text { (However based on the sheet which } \\
\text { they filled on adherence to following } \\
\text { the super brain yoga, } 8 \text { of the } \\
\text { participants could not be included } \\
\text { as their adherence was }<50 \% \text { of the } \\
\text { required period. Hence the complete } \\
\text { analysis and the result presented } \\
\text { here is of } 32 \text { participants only } \\
\text { (attrition rate-20\%) }\end{array}$ & Not information & Not information \\
\hline Lattie et al. [19] & Low risk & Low risk & Low risk & Low risk & Low risk & Low risk & Low risk \\
\hline $\begin{array}{l}\text { Rosenzweig } \\
\text { et al. [18] }\end{array}$ & Low risk & Low risk & Low risk & Low risk & Low risk & Low risk & Low risk \\
\hline $\begin{array}{l}\text { Hammer } \\
\text { et al. [32] }\end{array}$ & Low risk & Low risk & Low risk & Low risk & Low risk & Low risk & Low risk \\
\hline
\end{tabular}

persistence on unprocessed negative emotions, and the relationship between stressful events and sleep procession. At the end of the seminar, all participants were surveyed using a questionnaire.

\section{Cognitive and behavioral interventions}

Three studies reported cognitive and behavioral interventions [19], [26], [30]. In the first study, participants were invited to use an internet-based CBT program focused on mood management and symptom prevention for 16 weeks. At the end of the program, perceived stress, QoL, and the development of cognitive and behavioral coping skills were measured. The second study used 10-week positive psychological interventions (PPIs) to improve medical students' SWB, depression, and anxiety [26]. Participants underwent electroencephalogram (EEG) recordings before and after training to see if there was any frontal alpha EEG asymmetry.

The participants in the third study [30] received a cognitive-behavioral treatment protocol for neuroticism that was adapted into a single-session, preventive intervention, which has been considered a predictor of depressive and anxiety disorders. Experiential avoidance, QoL, self-esteem, empathy, and anxiety symptom scales were evaluated at baseline, 7 and 30 days after the intervention.

\section{Hypnosis}

One study reported using clinical hypnosis as an anxiety intervention for 1st-year medical students [32]. The intervention consisted of a 1-h individual session of hypnotherapy conducted by the same accredited hypnotherapist. The participants also received a hypnosis audio record that should be played once a day as an intervention at home.

\section{Life skill training}

Two studies used life skill training as their intervention to anxiety [25], [27]. The first was an 8-week training with a 2-h session weekly delivered over a course performed by participants under the supervision of two trained psychiatric post-graduates. The training approach included explaining the content, video-watching, roleplaying, group practice exercises, discussion, and homework assignments [25]. The second study [27] used well-being therapy measured using the Chinese version of Ryff's psychological well-being scales. The therapy was given in 5 sessions which consisted of psychological well-being education and practice that involved recording the circumstances surrounding some topics in a structured diary and implementing learned skills in daily life. In the fifth session, reflection on the implementation of these skills was explored.

\section{Assessment measures}

Most studies evaluated anxiety level changes after the psychotherapy intervention. The outcome was always measured using standardized tools or questionnaires, which vary significantly among the studies. Specific tools used to assess anxiety were GAD-7, DASS, The WTAS, SAI, BAI, SAS, The Spielberger State-Anxiety Inventory, The OASIS, SCL90 Anxiety Subscale, HADS-D, PAF - German version of TAI-G questionnaire, and The POMS. Besides anxiety and other psychiatric symptoms such as depression and psychological distress, several studies also measured QoL and well-being [19], [26], [27], [28], [30], [31], 
self-esteem or self-efficacy [16], [25], academic measures [28], [34], [35], and somatic symptoms [15], [19], [22], [23], [29]. No study exclusively focused on anxiety in medical students as all of the studies mentioned above explored more than one outcome.

\section{Outcomes}

\section{Psychoeducation and relaxation techniques}

Significant differences in anxiety levels were noticed in the groups who received relaxation exercises. In the experimental group, anxiety levels reduced after students went through relaxation techniques. The overall QoL was also significantly improved after 2 months of relaxation exercises [36]. Besides, there were increases in total self-efficacy coping, and in two of the self-efficacy subcategories: Stop unpleasant thoughts and problem-focused coping [16]. Decreased scores in the intervention group were also found in the test scores for psychological distress and motivation who received psychoeducation, progressive muscle relaxation, and systematic desensitization [21].

Students who did yoga to reduce anxiety reported improvement in general and mental well-being following the intervention [23] and a significant decrease in the anxiety level, shifting from a moderate level [22]. However, no significant heart rate change was found between the pre and post-test assessments [22].

Results showed a significant difference in the anxiety level after counseling between the experimental and control groups [24]. Meanwhile, no statistically significant differences were found between the experimental and control groups [33]. However, perceived medical school stress decreased in the coaching group and increased in the control group. This difference is statistically significant, yet the effect size is small.

\section{Cognitive and behavioral interventions}

Cognitive and behavioral interventions led to reduced anxiety symptoms [26], depression symptoms [26], [30], and a slight increase in the use of cognitive coping skills to reduce anxiety and depressive symptoms [19]. The cognitive-behavioral treatment protocol for neuroticism adapted into a single-session preventive intervention by Bermudez et al. [30] resulted in fewer students meeting the criteria for social anxiety disorders and panic disorder and improved QoL. Furthermore, PPIs as a form of cognitive and behavioral interventions were also able to promote SWB and prevent mental distress [30].

\section{Hypnosis}

A study by Hammer et al. [32] showed that students who received hypnosis had a reduction in the
PAF scores between the first survey before hypnosis (PAF-1) and the last survey (PAF-3). Furthermore, hypnosis has led to a statistically significant reduction in the lack of confidence subscale between PAF-1 and PAF-3 and a decline in the mean interference subscale score over time. The subscales were part of the PAF scores with a lack of confidence subscale measuring students' confidence in their abilities. In contrast, the interference subscales assessing irrelevant thoughts crossing students' minds during examinations.

Self-hypnosis is considered not to cause addiction, has no side effects and is a relatively safe alternative as an anxiety treatment. In economics, selfhypnosis is practical and quick to do an initial treatment. However, in complex and chronic cases, it may take longer. In patients who have serious problems, selfhypnosis can be combined with other treatments. The disadvantage of self-hypnosis is that many hypnosis services do not yet have a license [37].

\section{Mindfulness-based interventions}

Based on ten studies that used mindfulness as their intervention to reduce anxiety, four studies showed no significant outcomes. Neto et al. used a 6-weekmeditation course intervention in the large group, found no significant improvement of QoL, anxiety, stress, and depression in the intervention group [31]. McGrady et al. [15] also found no significant changes in anxiety or frequency of acute illness using mindfulness lessons, but there was a significant decrease in depression in the intervention group.

A study of students who joined a peer-support and mindfulness group did not significantly improve anxiety and depression symptoms and the QoL, resilience, or academic measures in the 8 weeks of the intervention group [28]. Another study using short-term breathing meditation practice showed no significant change in psychiatric symptoms, memory function, academic performance and achievement among normal, intelligent, and mentally healthy persons [35].

Significant results were found in six studies that used mindfulness as an intervention. Finkelstein et al. [17] showed a significant decrease in anxiety level and perceived stress regarding medical school pre- and post-intervention and decreased anxiety levels sustained for up to 3 months of evaluation after the intervention. Danilewitz et al. [34] showed significant preto-post-test changes in stress levels, self-compassion, altruism, two facets of mindfulness, and the program's positive impact on students' clinical performance in the intervention group. Warnecke et al. [29] also showed a significant reduction of stress and anxiety level in senior medical students after 8 weeks post-trial follow-up revealed that the effect was maintained after 8 weeks.

Based on Keng et al. [38], students reported significantly higher levels of trait mindfulness, subjective 
happiness and satisfaction with life, and lower levels of depressive symptoms, anxiety, general psychiatric symptoms, and perceived stress after the intervention. Rosenzweig et al. [18] also reported significantly less depression, less anxiety, greater empathy, a greater sense of spirituality, and a level of TMD post-intervention.

\section{Life skill training}

A significant reduction in SAS score in the intervention group compared to the control group was found in participants receiving cognitive-behavioral coping skills training program called William Life Skill Training [25]. In another study, compared with the control group, students undergoing the 5-week psychological well-being education and practice reported more extensive improvements in psychological well-being and adaptation and more significant alleviation in symptoms of anxiety and depression from pre-test to post-test to follow-up [27].

\section{Discussion}

This review has explored various interventions in reducing anxiety in medical students, including psychoeducation, relaxation techniques, cognitive and behavioral interventions, hypnosis, mindfulness program, and life skill training. Most of the studies used a mindfulness-based program. This approach emphasizes being aware of the experiences in the present moment in an intentional and non-judgmental manner. This review finds that in several studies, students who received mindfulness interventions reported lower levels of anxiety, depression, and stress and improvements in self-efficacy, self-compassion, altruism, and mindfulness [17], [18], [29], [34]. These changes were attributed to students' ability to cope with stress after undergoing the programs [17].

On the other hand, fewer studies found that mindfulnessprogramsdidnotchangeanxiety, depression, stress, QoL, memory function, academic performance, or academic achievement [15], [28], [31], [35]. Although students showed strong interest in participating in mindfulness programs, several studies reported poor program adherence due to difficulties juggling multiple medical school commitments. This might be one of the reasons that led to the discrepancies in the results of these studies. Many pieces of the literature suggest that mindfulness-based interventions may help alleviate mental health problems and improve psychological functioning.

This review grouped yoga, seminar, and counseling into one intervention group called psychoeducation and relaxation techniques. Almost all of the studies agreed that these techniques led to a reduction in anxiety levels. Only one study found no statistically significant changes in anxiety level but reported a lower level of perceived stress among students after a psychoeducative seminar and personal coaching sessions [33]. It is well understood that medical students face significant stress during their education journey from having to meet expectations, relentless competition, feeling of inadequacy to lack of time spent doing leisure activities, especially in the 1st year of medical school [6], [39]. This psychological distress often accumulates to manifest as anxiety and depressive symptoms. Reducing stress may eventually help to alleviate symptoms of anxiety and depression in medical students. This was evident in a study that experimented with yoga which found that yoga was an effective stress-reducing activity that reduces anxiety levels by balancing the autonomic nervous system measured by Heart Rate Variability [22]. Apart from improving anxiety levels, students have also reported that yoga helped them sleep better, allowed them to have better concentration in their studies and better anger management, helped them more relaxed throughout the day, and provided them with a boost of positive energy to start the day [23]. Besides yoga, relaxation techniques have also been proven to reduce anxiety in medical students. Relaxation techniques allowed students to learn better coping strategies when faced with anxiety-provoking situations [16]. Consequently, it helped students gain back their motivations that were often lost while struggling with anxiety and improved their QoL [21], [36].

Relaxation techniques reduce symptoms of anxiety and depression. Patients treated with relaxation techniques experienced reduced muscle stiffness and pain, better sleep habits, improved work performance and social functioning [40]. In addition, relaxation techniques have been shown to help older adults manage their anxiety. However, relaxation techniques should not be used as the chief strategy for treating patients with GAD. Studies showed better long-term improvements in patients treated with CBT than relaxation techniques. Therapeutic management as relaxation has been described to be helpful in the treatment of anxiety/depression. Here, anxiolytic effects of therapeutic management may occur by promoting inhibitory gamma-aminobutyric acidergic tone in specific areas of the brain. Thereby, nitric oxide and endocannabinoid pathways may also be involved [41].

An intervention that has long been studied and practiced to manage anxiety in medical students is CBT, which generally instills better coping skills. In this day and age, cognitive-behavioral interventions can be delivered through various means, from traditional face-to-face sessions to internet-based applications. This review achieved a reduction in anxiety symptoms through face-toface interventions but not by an internet-based CBT program [19], [26], [30]. It is interesting to note that 
the conflicting results might be due to the differing nature of the studies and the number of drop-out rates among participants. While face-to-face sessions were able to ensure better engagement, that was not the case with web-based applications, where it depended heavily on the willingness of participants to log in and use the applications. Although students initially were interested in joining the study, the actual number of students who used the program was low [19]. Besides, these studies have a different baseline characteristic with regard to the anxiety levels of the participants at the beginning of the study, which affected how many changes could be made after interventions. Another form of intervention similar to CBT is life skill training. Life Skill training includes instructions that teach students various coping skills [25]. The two studies using this intervention reported reduced anxiety symptoms [25], [27]. In William LifeSkill Training, medical students were trained to use a wide variety of cognitive-behavioral coping skills in an easy-to-deliver and cost-effective manner. These strategies aim to help students improve their self-esteem and provide them with social support. Improved self-esteem following the life skill training may play an active role in lessening medical students' anxiety and depression and improving mental health [25].

Meanwhile, well-being therapy involves experiencing deep connections with others, identifying potential, setting and pursuing goals, regulating demands and opportunities, and experiencing selfacceptance and self-determination. This therapy would enhance individuals' coping capacity and resilience, subsequently reducing negative emotions and preventing these emotions from accumulating into clinical disorders [27]. It is also believed that these pieces of training will have long-term benefits because students can continue to use the skills to cope with future stressful experiences.

An intervention that has yet been widely explored but shows potential in reducing anxiety in medical students is hypnosis. Hypnosis is one of the interventions that have yet to be explored by many. A study by Hammer et al. [32] reported that a 1-h hypnosis session helped reduce test anxiety in medical students. It is essential that this finding has to be taken with a pinch of salt as the study was a pilot project and, therefore, used a minimal sample size. It presents us with a possibility that hypnosis may help students to cope with anxiety before a test. Further studies need to be done to understand better the effect of hypnosis on anxiety levels among medical students with a much larger sample size and the mechanism that leads to the potential effects.

Hammer et al., test anxiety is an anxiety disorder often observed in students, especially medical students [42]. It is characterized by a fear of a performance/test situation, leading to specific cognitive, behavioral, and affective responses that may cause lower performance and failure in examinations [21], [43]. Several studies observed that examination-induced stress caused significant negative impacts on medical students' mental and general health [21], [33], [44]. Discrepancies in the results of the studies might be affected by the types of anxiety being observed [21], [32], [33].

Many studies have found that female medical students have a higher risk of suffering from stress and, subsequently, anxiety and depression than their male counterparts [10], [15], [36]. This is related to the lower self-perception of physical health and stress effects of academic activities among female medical students [45]. Female students perceived more significant stress than male students during examinations (especially multiplechoice examinations) when communicating with faculty staff and during autopsy [45], [46]. Obradović et al. [47] also observed that female students showed significant interpersonal sensitivity. Given how stress affects different gender, we need to be more careful when comparing interventional studies that targeted a specific gender as subjects. In this review, two studies that used single gender showed similar results regarding their interventions' effects on anxiety. Dehghan-Nayeri et al. [36] reported that 8-weeks of relaxation techniques lowered female students' anxiety levels, whereas Gurupriya and Mathangi [22] observed that 30-day super brain yoga decreased male students anxiety levels significantly.

Furthermore, all of the studies assessed various tools, such as tools to assess depression, stress, mood, and anxiety. This can lead to discrepancies between the author's intended objectives or outcomes (to reduce anxiety) and the tools used to evaluate anxiety. There needs further research about anxiety interventions using more precise tools, so the results are more consistent with the objectives research. Compared to a similar systematic review or meta-analysis, the review results confirm the systematic review and meta-analysis results. In a systematic review that performed subgroup analysis for common mental health problems among university and college students, it was found that CBT and mindfulness-based interventions were effective for both depression and GAD, and attention/perception modification was effective for GAD among university and college students in general. Other interventions, such as art, exercise, and peer support, had the most significant effect sizes for depression and GAD among college and university students [48].

In addition, this review has several limitations. In some studies, the control group did not receive any interventions. One of the studies [18] never mentioned the female: male ratio of the subjects involved. In six studies, there were no controls, so they only evaluated the intervention in one group and did not divide the group as a comparison. 


\section{Conclusion}

Psychotherapy may have an opportunity to decrease anxiety in medical students. Various interventions are carried out to decrease stress, depression, and anxiety in medical students, such as mindfulness-based intervention, psychoeducation and relaxation technique, cognitive and behavior intervention, hypnosis, and life skill training. The most effective and most likely gainful were found in cognitive and behavior intervention and mindfulness intervention. More research is needed on mental health interventions, especially on anxiety with precision tools and advanced assessment.

\section{References}

1. Boni RA, Paiva CE, de Oliveira MA, Lucchetti G, Fregnani JH, Paiva BS. Burnout among medical students during the first years of undergraduate school: Prevalence and associated factors. PLoS One. 2018;13(3):e0191746. PMid:29513668

2. Ghodasara SL, Davidson MA, Reich MS, Savoie CV, Rodgers SM. Assessing student mental health at the Vanderbilt University School of Medicine. Acad Med. 2011;86(1):116-21. PMid:21099385

3. Dyrbye LN, Thomas MR, Shanafelt TD. Systematic review of depression, anxiety, and other indicators of psychological distress among U.S. and Canadian medical students. Acad Med. 2006;81(4):354-73.

PMid: 16565188

4. Haldorsen $\mathrm{H}$, Bak NH, Dissing A, Petersson B. Stress and symptoms of depression among medical students at the University of Copenhagen. Scand J Public Health. 2014;42(1):89-95.

PMid:23999855

5. Bassols AM, Okabayashi LS, Silva AB, Carneiro BB, Feijó F, Guimarães GC, et al. First- and last-year medical students: Is there a difference in the prevalence and intensity of anxiety and depressive symptoms? Rev Bras Psiquiatr. 2014;36(3):233-40.

6. de Sousa JM, Moreira CA, Telles-Correia D. Anxiety, depression and academic performance: A study amongst Portuguese medical students versus non-medical students. Acta Med Port. 2018;31(9):454-62.

PMid:30332368

7. Chandavarkar U, Azzam A, Mathews CA. Anxiety symptoms and perceived performance in medical students. Depress Anxiety. 2007;24(2):103-11.

PMid:16845642

8. Patriquin MA, Mathew SJ. The neurobiological mechanisms of generalized anxiety disorder and chronic stress. Chronic stress (Thousand Oaks). 2017;1:2470547017703993. PMid:29503978

9. Kim KJ. Factors associated with medical student test anxiety in objective structured clinical examinations: A preliminary study. Int J Med Educ. 2017;7:424-7.

PMid:28035056

10. Ibrahim N, Al-Kharboush D, El-Khatib L, Al-Habib A, Asali D.
Prevalence and predictors of anxiety and depression among female medical students in King Abdulaziz University, Jeddah, Saudi Arabia. Iran J Public Health. 2013;42(7):726-36.

PMid:24427751

11. Cook AF, Arora VM, Rasinski KA, Curlin FA, Yoon JD. The prevalence of medical student mistreatment and its association with burnout. Acad Med. 2014;89(5):749-54.

PMid:24667503

12. Moriyama TS, Polanczyk G, Caye A, Banaschewski T, Brandeis D, Rohde LA. Evidence-based information on the clinical use of neurofeedback for ADHD. Neurotherapeutics. 2012;9(3):588-98.

PMid:22930416

13. Marzbani $H$, Marateb HR, Mansourian M. Neurofeedback: A comprehensive review on system design, methodology and clinical applications. Basic Clin Neurosci. 2016;7(2):143-58. PMid:27303609

14. Popay J, Roberts H, Sowden A, Petticrew M, Arai L, Rodgers M, et al. Guidance on the Conduct of Narrative Synthesis in Systematic Reviews. A Product from the ESRC Methods Programme; 2006. Available from: https://www.lancaster.ac.uk/ media/lancaster-university/content-assets/documents/fhm/dhr/ chir/NSsynthesisguidanceVersion1-April2006.pdf.

15. McGrady A, Brennan, J, Lynch D, Whearty K. A wellness program for first year medical students. Appl Psychophysiol Biofeedback. 2012;37(4):253-60.

PMid:22699926

16. Brennan J, McGrady A, Lynch DJ, Schaefer P, Whearty K. A stress management program for higher risk medical students: Preliminary findings. Appl Psychophysiol Biofeedback. 2016;41(3):301-5. PMid:26969177

17. Finkelstein C, Brownstein A, Scott C, Lan YL. Anxiety and stress reduction in medical education: An intervention. Med Educ. 2007;41(3):258-64.

PMid: 17316210

18. Rosenzweig S, Reibel DK, Greeson JM, Brainard GC Hojat M. Mindfulness-based stress reduction lowers psychological distress in medical students. Teach Learn Med. 2003;15(2):88-92.

PMid: 12708065

19. Lattie EG, Kashima K, Duffecy JL. An open trial of internet-based cognitive behavioral therapy for first year medical students. Internet Interv. 2019;18:100279.

PMid:31534911

20. Phang CK, Mukhtar F, Ibrahim N, Keng SL, Sidik SM. Effects of a brief mindfulness-based intervention program for stress management among medical students: The Mindful-Gym randomized controlled study. Adv Health Sci Educ Theory Pract. 2015;20(5):1115-34.

PMid:25697124

21. Saravanan C, Kingston R. A randomized control study of psychological intervention to reduce anxiety, amotivation and psychological distress among medical students. J Res Med Sci. 2014;19(5):391-7.

PMid:25097619

22. Gurupriya R, Mathangi DC. Effect of super brain yoga on heart rate variability among undergraduate medical students. Ann Trop Med Public Health. 2020;23(S13A):SP231313.

23. Bansal R, Gupta M, Agarwal B, Sharma S. Impact of short term yoga intervention on mental well being of medical students posted in community medicine: A pilot study. Indian J Community Med. 2013;38(2):105-8.

PMid:23878424 
24. Velayudhan A, Gayatridevi S, Bhattacharjee R. Efficacy of behavioral intervention in reducing anxiety and depression among medical students. Ind Psychiatry J. 2010;19(1):41-6. PMid:21694790

25. Li C, Chu F, Wang H, Wang XP. Efficacy of Williams LifeSkills training for improving psychological health: A pilot comparison study of Chinese medical students. Asia Pac Psychiatry. 2014;6(2):161-9.

PMid:23857943

26. Xu YY, Feng ZQ, Xie YJ, Zhang J, Peng SH, Yu YJ, et al. Frontal alpha EEG asymmetry before and after positive psychological interventions for medical students. Front Psychiatry. 2018;9:432. PMid:30254589

27. $\mathrm{Xu} Y \mathrm{Y}, \mathrm{Wu}, \mathrm{T}, \mathrm{Yu} \mathrm{YJ}, \mathrm{Li} \mathrm{M}$. A randomized controlled trial of well-being therapy to promote adaptation and alleviate emotional distress among medical freshmen. BMC Med Educ. 2019;19(1):182.

28. Moir F, Henning M, Hassed C, Moyes SA, Elley CR. A peersupport and mindfulness program to improve the mental health of medical students. Teach Learn Med. 2016;28(3):293-302. PMid:27092397

29. Warnecke E, Quinn S, Ogden K, Towle N, Nelson MR. A randomised controlled trial of the effects of mindfulness practice on medical student stress levels. Med Educ. 2011;45(4):381-8. PMid:21401686

30. Bermudez MB, Costanzi M, Macedo MJ, Tatton-Ramos T, Xavier AC, Ferrão YA, et al. Improved quality of life and reduced depressive symptoms in medical students after a single-session intervention. Braz J Psychiatry. 2019;42(2):145-52. PMid:31859792

31. Neto AD, Lucchetti AL, Ezequiel OS, Lucchetti G. Effects of a required large-group mindfulness meditation course on first-year medical students' mental health and quality of life: A randomized controlled trial. J Gen Intern Med. 2020;35(3):672-8. PMid:31452038

32. Hammer CM, Scholz M, Bischofsberger $L$, Paulsen $F$ Burger $\mathrm{PH}$. Feasibility of clinical hypnosis for test anxiety in firstyear medical students. Int J Clin Exp Hypn. 2020;68(4):511-20. PMid:32804002

33. Kötter T, Niebuhr F. Resource-oriented coaching for reduction of examination-related stress in medical students: An exploratory randomized controlled trial. Adv Med Educ Pract. 2016;7:497-504

PMid:27601935

34. Danilewitz M, Bradwejn J, Koszycki D. A pilot feasibility study of a peer-led mindfulness program for medical students. Can Med Educ J. 2016;7(1):e31-7. PMid:27103950

35. Paholpak S, Piyavhatkul N, Rangseekajee P, Krisanaprakornkit T, Arunpongpaisal S, Pajanasoontorn $\mathrm{N}$, et al. Breathing meditation by medical students at Khon Kaen University: Effect on psychiatric symptoms, memory, intelligence and academic achievement. J Med Assoc Thai. 2012;95(3):461-9.

PMid:22550848
36. Dehghan-Nayeri N, Adib-Hajbaghery M. Effects of progressive relaxation on anxiety and quality of life in female students: A non-randomized controlled trial. Complement Ther Med. 2011;19(4):194-200.

PMid:21827933

37. Hammond DC. Hypnosis in the treatment of anxiety- and stressrelated disorders. Expert Rev Neurother. 2010;10(2):263-73. PMid:20136382

38. Keng SL, Phang CK, Oei TP. Effects of a brief mindfulnessbased intervention program on psychological symptoms and well-being among medical students in Malaysia: A controlled study. Int J Cognitive Ther. 2015;8(4):335-50.

39. Patnaik A, Choudhury KC. Assessment of risk factors associated with hypertension among undergraduate medical students in a medical college in Odisha. Adv Biomed Res. 2015;4:38. PMid:25789264

40. Ali $U$, Hasan $S$. The effectiveness of relaxation therapy in the reduction of anxiety related symptoms (a case study). Int $\mathrm{J}$ Psychol Stud. 2010;2(2):202-8.

41. Esch T, Fricchione GL, Stefano GB. The therapeutic use of the relaxation response in stress-related diseases. Med Sci Monit. 2003;9(2):RA23-34.

PMid: 12601303

42. Schaefer A, Matthess H, Pfitzer G, Köhle K. Mental health and performance of medical students with high and low test anxiety. Psychother Psychosom Med Psychol. 2007;57(7):289-97. PMid: 17357900

43. Farooqi YN, Ghani R, Spielberger CD. Gender differences in test anxiety and academic performance of medical students. Int J Psychol Behav Sci. 2012;2(2):38-43.

44. Lyndon MP, Strom JM, Alyami HM, Yu TC, Wilson NC, Singh PP, et al. The relationship between academic assessment and psychological distress among medical students: A systematic review. Perspect Med Educ. 2014;3(6):405-18. PMid:25428333

45. Backović DV, Zivojinović Jl, Maksimović J, Maksimović M Gender differences in academic stress and burnout among medical students in final years of education. Psychiatr Danub. 2012;24(2):175-81. PMid:22706416

46. Kipping CJ. Stress in mental health nursing. Int J Nurs Stud. 2000;37(3):207-18. PMid: 10754186

47. Obradović J, Long JD, Cutuli JJ, Chan CK, Hinz E, Heistad D, et al. Academic achievement of homeless and highly mobile children in an urban school district: Longitudinal evidence on risk, growth, and resilience. Dev Psychopathol. 2009;21(2):493-518. PMid:19338695

48. Huang J, Nigatu YT, Smail-Crevier R, Zhang X, Wang J Interventions for common mental health problems among university and college students: A systematic review and meta-analysis of randomized controlled trials. J Psychiatr Res. 2018;107:1-10.

PMid:30300732 